\title{
The SARS-CoV-2 receptor, ACE-2, is expressed on many different cell types: implications for ACE-inhibitor- and angiotensin II receptor blocker-based antihypertensive therapies-reply
}

\author{
Adriana Albini $^{1}\left[\right.$ ] Douglas McClain Noonan ${ }^{1,2} \cdot$ Giuseppe Pelosi $^{3,4} \cdot$ Giovanni Di Guardo $^{5} \cdot$ Michele Lombardo $^{6}$
}

Received: 27 June 2020 / Accepted: 6 July 2020 / Published online: 14 July 2020

(c) Società Italiana di Medicina Interna (SIMI) 2020

\section{Dear Editor,}

We read with great interest the comment by Dr. De Cauwer [1] in response to our Point of View [2]. He raises several interesting points.

Chromosome $\mathrm{X}$ harbors the gene coding for angiotensin converting enzyme 2 (ACE-2) receptor, which allows SARSCoV-2 entry into host cells. This could partially explain, in turn, the increased mortality reported in males in comparison with females: indeed, deaths attributed to SARS-CoV-2 infection were significantly higher among male individuals, both in China and in Italy [2]. Males carrying rare ACE2-coding variants (polymorphisms) will express those variants in all ACE-2-expressing cells, whereas females will typically express those variants in a mosaic distribution, driven by early $\mathrm{X}$-inactivation events. The aggregated prevalence of the aforementioned ACE-2 variants is supposed to be 3.9 per 1000 males and 8.5 per 1000 females [3]. If this holds true, it might indirectly suggest a protective role of ACE-2 overexpression in the clinical course of SARS-CoV-2 infection in female patients. The SARS virus in infected cells down-regulates the ACE-2, we mentioned this in our Point

Adriana Albini

adriana.albini@multimedica.it

1 Scientific and Technology Pole, IRCCS MultiMedica, Milan, Italy

2 Department of Biotechnology and Life Sciences, University of Insubria, Varese, Italy

3 Department of Oncology and Hemato-Oncology, University of Milan, Milan, Italy

4 Inter-Hospital Pathology Division, IRCCS MultiMedica, Milan, Italy

5 University of Teramo, Faculty of Veterinary Medicine, 64100 Teramo, Italy

6 Cardiology Unit, San Giuseppe Hospital-MultiMedica, Milan, Italy of View [2]. ACE-2 produces Ang (1-7) heptapeptide, an anti-inflammatory molecule.

Zoonotic human beta-coronaviruses (SARS-CoV-1, MERS and SARS-CoV-2) have multiple strategies for proteolytic activation of spike (S) protein and viral entry, including cell surface transmembrane protease/serine (TMPRSS) proteases, endosomal cathepsins, and furin [2, 4]. The transmembrane protease serine 2 TMPRSS2, an androgendependent enzyme, acts in reinforcing the ACE-2 receptor activity in allowing cell entry to a number of viral pathogens as well as to SARS-CoV-2 as reported in our Point of View [2]. Moreover, the expression of TMPRSS2 on endothelial cells together with ACE- 2 could account for the severity of SARS-CoV-2 infection via microthrombus formation beyond pro-inflammatory cytokines release causing a multiorgan failure alongside a disseminated intravascular coagulation [2]. The androgen receptor (AR) gene and ACE-2 both map to chromosome $\mathrm{X}$ and polymorphisms of these genes could result in different biological effects. The low androgen levels in females could be inferred in reducing the synergistic activity of TMPRSS 2 with ACE-2 Receptor. As a consequence, a minor load of SARS-CoV-2 virions could colonize the lung, heart and vascular system, as well as all the other ACE-2-expressing cells and tissues throughout the human body. This could pave the way to a new therapeutic target potentially counteracting SARS-CoV-2 infection. It would be interesting to test the effect of currently available protease inhibitors, eventually associated with renin-angiotensin-aldosterone system (RAAS)-inhibitors, in an attempt to reduce, if not even inhibit viral entry into susceptible host cells [2]. This will be mitigating SARS-CoV-2-induced cardiovascular, respiratory, enteric, renal, hepatic and cerebral damages by means of the protective role exerted by the increased amounts of Ang (1-7) [2].

The gene coding for TMPRSS2 is located on chromosome 21, as mentioned in the comment of Dr De Cauwer [1]. Individuals with trisomy 21 already show, by themselves, clear 
signs of inflammation, along with an increased frequency of autoimmune disease conditions [5]. People with trisomy 21 syndrome are additionally known to experience many different signs of chronic immune dysregulation and a propensity to develop clinical pictures consistent with a "perfect immunologic storm", most likely resulting in a greater severity also of SARS-CoV-2 infection [5]. Specifically, this would be driven by a sudden and rapid increase of cytokine and chemokine release, resulting in a higher susceptibility to develop clinically relevant respiratory infections, both bacterial and viral $[1,5]$. The negative impact of the "cytokine storm" in SARS-CoV-infected subjects is driven by a cascade of pathophysiological events involving acute respiratory distress, myocardial injury and vascular damage with thromboembolism and DIC. Furthermore, on chromosome 21 , there are four out of the six known interferon gene receptors, among which the two type I IFN receptors IFNAR1 and IFNAR2, along with the type II IFN receptor IFNGR2 [5].

As far as concerns the greater clinical disease severity of CoViD-19 in trisomy 21-affected patients, De Cauwer et al. have reported 4 female subjects with Down syndrome, aged between 48 and 62 years, whose clinical course of SARS-CoV-2 infection was particularly severe, with an acute respiratory distress syndrome warranting hospital care in three and a fatal outcome in one patient [1]. Yet in trisomy 21 individuals a TMPRSS2 protease overexpression has been documented, as mentioned in the comment of Dr De Cauwer [1], with this leading to an increased viral infection's rate of susceptible host's cells and tissues. This could be of concern also for SARS-CoV-2 infection, thus justifying a new therapeutic approach based upon protease inhibitors, like camostat mesylate, nafamostat mesylate or bromhexine chlorhydrate (a mucolytic agent marketed in 1963) eventually combined to RAAS antagonist drugs, or to anti-inflammatory drugs. However, also in the case of trisomy 21 syndrome [5], we need adequately powered studies to compare the clinical course of SARS-CoV-2 infection in age and gender-matched patients in comparison to the general population.

In conclusion, the interesting comment by $\mathrm{Dr} \mathrm{De} \mathrm{Cau}-$ wer points out the complex interactions between ACE-2 and TMPRSS2 with reference to the clinical course of CoViD-19 as well as to SARS-CoV-2 infection's pathogenic evolution and severity degree in given population segments of infected individuals, like male individuals and Down syndromeaffected patients [1]. In this regard, the interplay between RAAS-inhibiting drugs and the various disease phenotypes of SARS-CoV-2 infection underscores the need of clarifying the relevance of such relationships from a clinic-pathological standpoint. We think that the hitherto available studies are, at the moment, insufficient to answer these relevant questions, which is substantially due to their "retrospective approach". Prospective, randomized, case-control studies will be able to define the therapeutic relevance and the clinical influence of the different classes of RAAS-antagonist drugs (ACE-Is, ARBs) on CoViD-19-affected patients, both in the general population as well as in subgroups stratified by age, gender and relevant cardiovascular conditions, such as diabetes, hypertension, ischemic heart disease, atrial fibrillation and finally, in patients affected by Down syndrome.

\section{Compliance with ethical standards}

Conflict of interest The authors declare that they have no conflict of interest.

Statement of human and animal rights This article does not contain any studies with human participants or animals performed by any of the authors.

Informed consent Informed consent was not required for this type of publication.

\section{References}

1. De Cauwer H (2020) The SARS-CoV-2 receptor, ACE-2, is expressed on many different cell types: implications for ACEinhibitor- and angiotensin II receptor blocker-based cardiovascular therapies: comment. Intern Emerg Med. https://doi.org/10.1007/ s11739-020-02406-Z

2. Albini A, Di Guardo G, Noonan DM, Lombardo M (2020) The SARS-CoV-2 receptor, ACE-2, is expressed on many different cell types: implications for ACE-inhibitor- and angiotensin II receptor blocker-based cardiovascular therapies. Intern Emerg Med. https ://doi.org/10.1007/s11739-020-02364-6

3. Gibson WT, Evans DM, An J, Jones SJ (2020) ACE 2 coding variants: a potential X-linked risk factor for COVID-19 disease. Science. https://doi.org/10.1101/2020.04.05.026633

4. Millet JK, Whittaker GR (2015) Host cell proteases: critical determinants of coronavirus tropism and pathogenesis. Virus Res 202:120-134. https://doi.org/10.1016/j.virusres.2014.11.021

5. Espinosa JM (2020) Down syndrome and COVID-19: a perfect storm? Cell Rep Med 1:100019. https://doi.org/10.1016/j. xcrm.2020.100019

Publisher's Note Springer Nature remains neutral with regard to jurisdictional claims in published maps and institutional affiliations. 